\title{
Purification of duck growth hormone and cloning of the complementary DNA
}

\author{
Huang-Tsu Chen ${ }^{a}$, Fu-Ming Pan ${ }^{\text {a,b }}$ and Wen-Chang Chang a,b \\ ${ }^{a}$ Institute of Biochemical Sciences, National Taiwan University, and ${ }^{b}$ Institute of Biological Chemistry, Academia Sinica, \\ Taipei (Taiwan, China)
}

(Received 21 August 1987)

(Revised manuscript received 26 October 1987)

Key words: Growth hormone; cDNA sequence; Amino acid sequence; Sequence homology; (Duck)

Duck growth hormone (GH) was isolated and purified from duck pituitaries by salt precipitation and HPLC on reverse-phase $C_{18}$ columns. The duck GH was homogeneous as shown by SDS-polyacrylamide gel electrophoresis with a molecular weight of 22000 . The cDNA was synthesized and cloned in Escherichia coli using EcoRI linkers and pBR322 as vector. The positive clones were selected and sequenced. The full-length duck GH cDNA contains 820 nucleotide pairs with an open reading frame coding for the precursor form duck GH of 216 amino-acid residues. The partial amino-acid sequence from the protein completely agrees with that derived from the cDNA, with Phe as the first residue in mature duck GH preceded by a 27-residue hydrophobic signal peptide. The duck GH is almost completely homologous to the chicken GH, with only three conservative substitutions (Ser for Thr, His for Tyr and Lys for Arg) and one deletion (Ala) in the duck GH sequence. Comparison of amino-acid sequence of duck GH with that of various species reveals $56 \%, 73 \%$ and $40 \%$ homologies with GHs of human, rat and salmon, respectively.

Growth hormone $(\mathrm{GH})$ of the vertebrate, a 22 $\mathrm{kDa}$ protein hormone synthesized in anterior pituitary gland, is required for preadult growth $[1,2]$. The GHs of many mammalian species have been well studied at both protein and gene levels. However, the avian GH has been only partially characterized at the protein level $[3,4]$. Although cDNA of chicken $\mathrm{GH}$ was cloned and characterized recently [5], the primary structures of other avian GHs remain unknown. In order to determine the amino-acid sequence of the duck $\mathrm{GH}$ and to provide the basis for further genomic studies, we have purified and characterized duck GH from pituitary glands and also synthesized,

Abbreviation: $\mathrm{GH}$, growth hormone.

Correspondence: W.C. Chang, Institute of Biological Chemistry, Academia Sinica, P.O. Box 23-106, Taipei, Taiwan, R.O.C. cloned and sequenced its cDNA. The results are described in this report.

Fresh pituitary glands were obtained from 3week-old mule ducks, a hybrid species between Kaiya duck (Anas platyrhynchos variant domestica) and muscovy drake (Cairina moschata). Duck $\mathrm{GH}$ was extracted with $50 \mathrm{mM}$ ammonium bicarbonate/ $20 \mathrm{mM}$ phenylmethanesulfonyl fluoride and then directly purified by reverse-phase HPLC using a $\mathrm{C}_{18}$ column and $0.1 \%$ TFA in water/0.1 $\mathrm{M}$ TFA in acetonitrile as solvent. The purified duck GH was confirmed for immunoreactivity and purity by Ouchterlony agar diffusion [11] and SDS-polyacrylamide gel electrophoresis, respectively. The amino-acid sequence was determined in a pulse-liquid phase protein sequencer (Applied Biosystems Inc. 477A).

The mRNA of the duck pituitary glands was purified and cloned as described previously [6]. Briefly, total RNA was extracted from the fresh 
pituitary glands by guanidine and hot phenol. Poly $(\mathrm{A})^{+}$RNA was selected by passing through the oligo-(dT)cellulose column twice. The $\operatorname{poly}(\mathrm{A})^{+} \mathrm{RNA}$ was used in the rabbit reticulocyte lysate in vitro translation system as well as in the hybridization test using ${ }^{32} \mathrm{P}$-labelled porcine $\mathrm{GH}$ cDNA as probe. The biologically active mRNA was used to construct a cDNA library using pBR322 in E. coli HB101 as host. The doublestranded cDNA was methylated with EcoRI

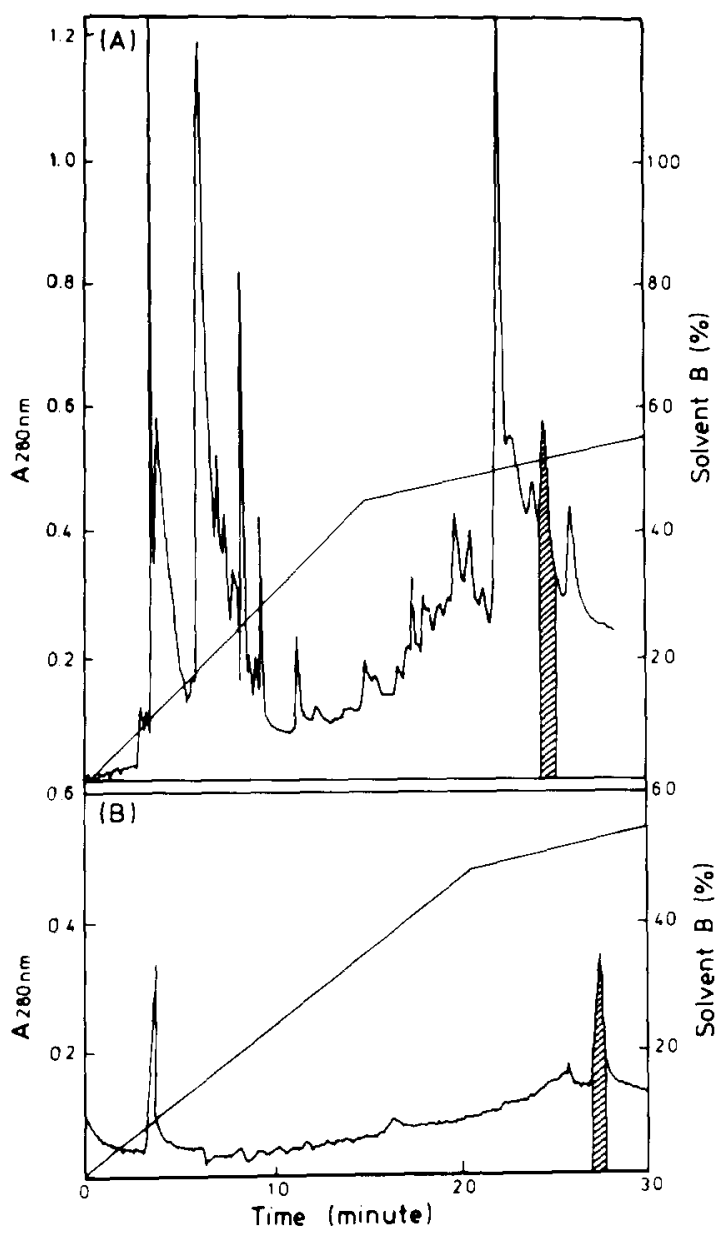

Fig. 1. (A) HPLC profile of duck pituitary extracts through a $\mathrm{C}_{18}$ column. (B) Rechromatography of duck $\mathrm{GH}$ from (A). Solvents were: (A) $0.1 \%$ trifluoroacetic acid in water; (B) $0.1 \%$ trifluoroacetic acid in acetonitrile. The hatched peaks in (A) and $(B)$ were found to contain duck $G H$ by the Ouchterlony agar diffusion technique [11] using monkey antiserum against porcine GH. The duck GH could form a precipitin line with this antiserum.

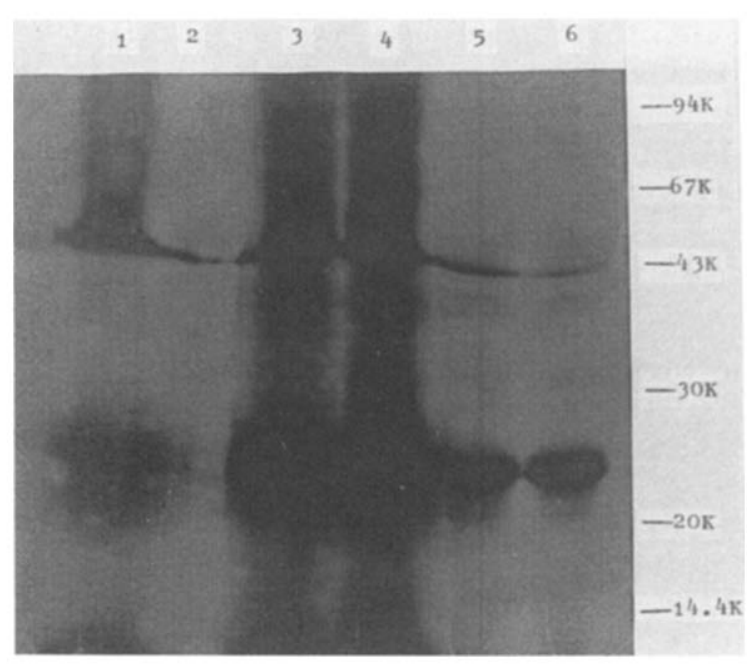

Fig. 2. SDS-polyacrylamide gel electrophoresis of the immunoprecipitated in-vitro-translated duck $\mathrm{GH}$. The duck pituitary mRNA was translated in vitro in rabbit reticulocyte lysate containing $\left[{ }^{35} \mathrm{~S}\right]$ methionine, and the products were precipitated with porcine $\mathrm{GH}$ antiserum. The immunoprecipitate was analyzed by SDS-polyacrylamide gel electrophoresis. Lane 1: control (without exogenous mRNA antiserum). Lane 2: control (without exogenous mRNA, but precipitated with antiserum). Lanes 3, 4: with duck mRNA, but without antiserum. Lanes 5,

6: with duck mRNA and precipitated with antiserum.
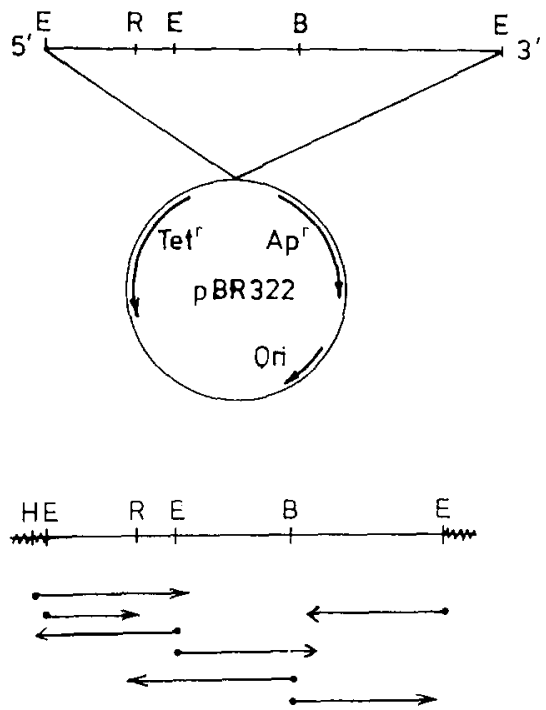

Fig. 3. Orientation of duck GH-cDNA in pBR322 and the sequencing strategy. B, Bam HI; E, Eco RI; H, HindIII; R, RsaI. The wavy lines indicate the sequence from pBR322. 
methylase before ligation with the EcoRI-treated vector.

Positive clones were selected by hybridization with ${ }^{32}$ P-labelled porcine GH-cDNA. The nucleotide sequence of the duck GH-cDNA was determined by the method of Maxam and Gilbert [7].

Two cycles of HPLC on a $\mathrm{C}_{18}$ column (Fig. 1) gave a duck $\mathrm{GH}$ preparation which could form a precipitin line with a monkey antiserum against porcine $\mathrm{GH}$ (data not shown). The $\mathrm{N}$-terminal amino-acid sequence was determined with an automatic protein sequencer as shown below (in one-letter code):

$\mathrm{H}_{2}$ N-FPAMPLSNLF ANAVLRAQH L -...
This sequence is identical to that of chicken GH [5] except that the latter contains a Thr preceding the first Phe residue.

The duck pituitary mRNA could direct the synthesis of a protein of about $25 \mathrm{kDa}$ (Fig. 2) in the rabbit reticulocyte lysate in vitro translation system. This protein must be the precursor form of duck $\mathrm{GH}$, as it could be recognized by the monkey antiserum against porcine $\mathrm{GH}$ in the immunoprecipitation test. The molecular size of this precursor duck $\mathrm{GH}$ is in agreement with that derived from the cDNA sequence (Fig. 4). In addition to the intense major band of $25 \mathrm{kDa}$ (Fig. 2), a band of $43 \mathrm{kDa}$ is also visible, which is most likely due to non-specific binding with the antiserum, since this band is also present in the control (lane 2).

$\mathrm{CC}$ 50 CCTTCAAGCAACATCTGAGCAACTCTCCAGGCAGAAATGGCTCCAGGGTCGTGGTTTTCTCCTCTCTTCATC 100 ACTGTGATCACCCTGGGATTGCAGTGGCCACAAGAAGCTGCCACCTTCCCAGCCATGCCCCTTTCCAACCTG $\begin{array}{llllllllllllllllllllllll}\text { T } & \text { V } & \text { I } & \text { T } & \text { L } & \text { G } & \text { L } & Q & \text { W } & \text { P } & \text { Q } & \text { E } & \text { A } & \text { A } & \text { T } & \text { F } & \text { P } & \text { A } & \text { M } & \text { P } & \text { L } & \text { S } & \text { N } & \text { L }\end{array}$ 150 200 TTTGCCAACGCTGTGCTGAGGGCTCAGCACCTCCACCTCCTGGCCGCAGAGACGTACAAAGAGTTCGAACGC $\begin{array}{lllllllllllllllllllllllll} & \text { A } & A & N & A & V & L & R & A & Q & H & L & H & \text { L } & \text { L } & \text { A } & \text { A } & \text { E } & \text { T } & \text { Y } & \text { H } & \text { E } & \text { F } & \text { E } & \text { R }\end{array}$ 250 AGCTATATTCCGGAGGACCAGAGACACACCAACAAGAATTCCCAGGCATTCTGTTACTCAGAAACCATCCCA $\begin{array}{lllllllllllllllllllllllll}\mathbf{S} & \mathbf{Y} & \mathrm{I} & \mathbf{P} & \mathbf{E} & \mathbf{D} & \mathbf{Q} & \mathbf{R} & \mathbf{H} & \mathbf{T} & \mathbf{N} & \mathbf{K} & \mathbf{N} & \mathbf{S} & \mathbf{Q} & \mathrm{A} & \mathrm{F} & \boldsymbol{C} & \mathrm{Y} & \mathbf{S} & \mathbf{E} & \mathrm{T} & \mathrm{I} & \mathrm{P}\end{array}$ 300 . 350

GCTCCCACAGGGAAGGATGATGCCCAGCAGAAATCAGATATGGAGCTTCTTCGGTTTTCACTGGTTCTCATC $\begin{array}{llllllllllllllllllllllll}\mathbf{A} & \mathrm{P} & \mathrm{T} & \mathrm{G} & \mathrm{K} & \mathrm{D} & \mathrm{D} & \mathrm{A} & \mathbf{Q} & \mathbf{Q} & \mathbf{K} & \mathrm{S} & \mathrm{D} & \mathrm{M} & \mathbf{E} & \mathrm{L} & \mathrm{L} & \mathbf{R} & \mathbf{F} & \mathbf{S} & \mathrm{L} & \mathrm{V} & \mathrm{L} & \mathbf{I}\end{array}$ CAGTCCTGGCTGACCCCAGTGCAATACCTAAGCAAGGTGTTTACAAACAACCTGGTTTTTGGCACCTCAGAC

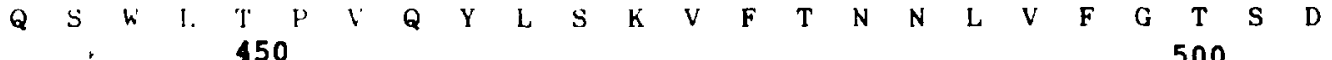
AGAGTGTTTGAAAAACTAAAGGACCTAGAAGAAGGGATCCAAGCTCTGATGAGGGAGCTGGAGGACCGGAGC $\begin{array}{llllllllllllllllllllllll}R & V & F & E & K & L & K & D & L & E & E & G & I & Q & A & L & M & R & E & L & E & D & R & S\end{array}$ CCGCGGGGCCCGCAACTCCTCAAGCCCACCTACGACAAGTTCGACATCCACCTGCGCAACGAGGACGCCCTG

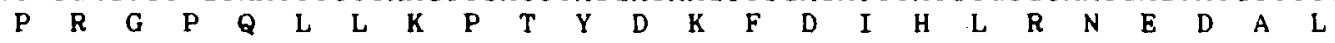
600 CTGAAGAACTACGGCCTGCTCTCCTGCTTCAAGAAGGACCTGCACAAGGTGGAGACCTACCTGAAGGTGATG

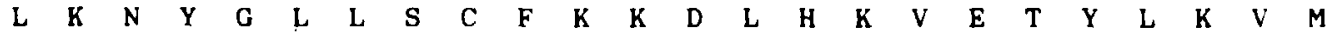
AAGTGCCGCCGCTTCGGAGAGAGCAACTGCACCATCTGAGGCCGGGACGGCCGCCCTCCATCCCGGCCCTCG $\begin{array}{lllllllllllll}\mathbf{K} & \mathbf{C} & \mathbf{R} & \mathbf{R} & \mathbf{F} & \mathrm{G} & \mathrm{E} & \mathbf{S} & \mathrm{N} & \mathbf{C} & \mathrm{T} & \mathrm{I}\end{array}$ 750 CCGCGGGCCTGCCCCGTCCCCTCTCACGAAAATCGCTCTGCCCTGGGGAGAATAAACCCGCTACCGCTGAGC

\section{AAAAAAAAAAAAAAAAAAAAAAAAA}

Fig. 4. Nucleotide sequence of duck GH-cDNA and the derived amino-acid sequence of duck GH with a calculated molecular weight of 24849 for the precursor form. 
The GH-encoding cDNA constitutes about 4\% of the total cDNA library, as calculated from the Southern hybridization study (data not shown). The high percentage would indicate that the GHencoding mRNA is one of the most abundant species, and this is expected for the rapidly growing ducks. The majority of the GH-encoding cDNAs contain around 800 nucleotide pairs and therefore appear to be full-length. In fact, in the nucleotide sequence of one of these clones (Fig. 4), the sequence flanking the initiator ATG codon is consistent with the consensus sequence (purineXXATGG) for eucaryotic initiation sites of translation [12]. Besides, this sequence encodes a typical GH precursor highly homologous to the $\mathrm{GH}$ of chicken as well as other vertebrates (Fig. 5). There seems little doubt that it is a full-length cDNA.

The duck GH-cDNA was sequenced as shown in Figs. 3 and 4. The amino-acid sequence of the duck GH derived from the nucleotide sequence of its cDNA is also shown in Fig. 4. As is evident from Fig. 5, both avian GHs differ at only four positions, including three conservative substitutions (Ser, His and Lys in duck GH vs. Thr, Tyr and $\mathrm{Arg}$ in chicken $\mathrm{GH}$, respectively) and one deletion of Ala in the duck GH. Although the cDNA of chicken $\mathrm{GH}$ has been cloned, its nucleotide sequence was not reported [5]. The homology between the GHs of duck and chicken at the cDNA level cannot be evaluated at present. The amino-terminal amino-acid sequence as determined by the protein sequencer confirms the first 20-amino-acid residues derived from the nucleotide sequence. It also elucidates that the mature duck GH starts with a unique amino acid, Phe, after cleavage of the 27-residue signal peptide. The signal sequence of duck $\mathrm{GH}$ is about the same size as that of most mammalian GHs (Fig. 5), but longer than the signal peptide of 22 residues of the Salmon GH [10]. Comparison of the GHs from several representative vertebrate species (Fig. 5) reveals that duck GH shares $56 \%, 73 \%$ and $40 \%$

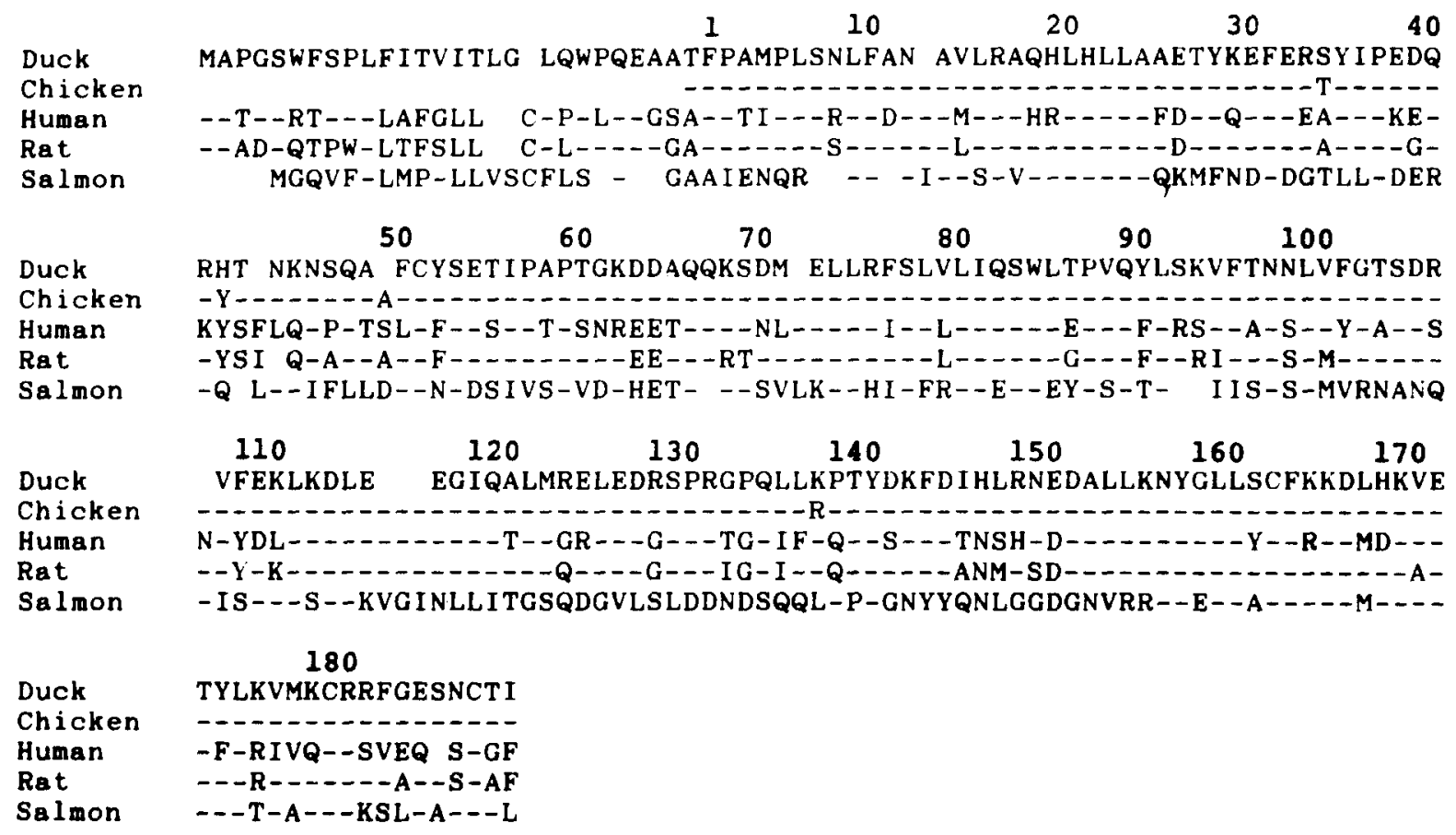

Fig. 5. Comparison of GHs from duck (present study), chicken [5], human [8], rat [9] and salmon [10]. The numbering begins from the first amino-acid residue in the mature hormone. Bars represent identical residues with duck GH. Blank spaces are introduced to maximize homology. 
sequence homology with the GHs of human, rat and salmon, respectively. Similar homology is found also when the coding region of duck GH cDNA is compared with that of other representative vertebrate species, except salmon; the duck GH shows $53 \%, 75 \%$ and $53 \%$ nucleotide-sequence homology with that of human, rat and salmon, respectively. It is interesting to note that duck GH exhibits much higher sequence homology with that of salmon at the cDNA level than at the protein level (53\% vs. $40 \%$ ). The significance of this difference is not clear at present.

\section{References}

1 Miller, W.L. and Eberhardt, N.L. (1983) Endocr. Rev. 4, 97-130.

2 Chawla, R.K., Parks, J.S. and Rudman, D. (1983) Annu. Rev. Med. 34, 519-547.
3 Farmer, S.W., Papkoff, H. and Hayashida, T. (1974) Endocrinology 95, 1560-1565.

4 Harvey, S. and Scanes, C.G. (1977) J. Endocrinol. 73, 321-329.

5 Souza, L.M., Boone, T.C., Murdock, D., Langley, K., Wypych, J., Fenton, D., Johnson, S., Lai, P.H., Everett, R., Hsu, R.Y. and Bosselman, R. (1984) J. Expt. Zool. 232, 465-473.

6 Chang, T. and Chang, W.C. (1987) Biochim. Biophys. Acta 910, 89-92.

7 Maxam, A.M. and Gilbert, W. (1977) Proc. Natl. Acad. Sci. USA 74, 560-564.

8 Martial, J.A., Hallewell, R.A., Baxter, J.D. and Goodman, H.M. (1979) Science 205, 602-607.

9 Seeburg, P.H., Shine, J., Martial, J.A., Baxter, J.D. and Goodman, H.M. (1977) Nature 270, 486-494.

10 Sekine, S., Mizukami, T., Nishi, T., Kuwana, Y., Saito, A., Sato, M., Itoh, S. and Kawauchi, H. (1985) Proc. Natl. Acad. Sci. USA 82, 4306-4310.

11 Ouchterlony, O. (1953) Acta Pathol. Microbiol. Scan. 32, 231-240.

12 Kozak, M. (1981) Nucleic Acids Res. 9, 5233-5252. 